\title{
Estimates of the prevalence of and current treatment practices for rheumatoid arthritis in Japan using reimbursement data from health insurance societies and the IORRA cohort (I)
}

\author{
Hisashi Yamanaka • Naonobu Sugiyama • \\ Eisuke Inoue $\cdot$ Atsuo Taniguchi $\cdot$ Shigeki Momohara
}

Received: 20 August 2012/ Accepted: 5 March 2013

(C) Japan College of Rheumatology 2013

\begin{abstract}
Objectives The prevalence of rheumatoid arthritis (RA) and its current treatment practices in Japan are poorly documented. Therefore, we examined these factors in a Japanese health insurance database.

Methods We analyzed reimbursement data provided by health insurance societies for 1 million individuals, including healthy individuals, registered from January 2005 to June 2011. Changes in treatments were determined in 320 thousand individuals originally registered in 2005 . The treatment patterns were compared with those of the Institute of Rheumatology, Rheumatoid Arthritis (IORRA) cohort managed by Tokyo Women's Medical University. Results The estimated prevalence of RA was 1.24 million (1.0\% of the Japanese population), excluding suspected cases, and 706 thousand $(0.6 \%)$ in a sensitivity analysis. Seventy-nine percent of patients were treated for RA.
\end{abstract}

Electronic supplementary material The online version of this article (doi:10.1007/s10165-013-0863-6) contains supplementary material, which is available to authorized users.

H. Yamanaka $(\varangle) \cdot$ E. Inoue $\cdot$ A. Taniguchi $\cdot$ S. Momohara Institute of Rheumatology, Tokyo Women's Medical University, 10-22 Kawada-cho, Shinjuku-ku, Tokyo 162-0054, Japan

e-mail: yamanaka@ior.twmu.ac.jp

E. Inoue

e-mail: einoue@kde.biglobe.ne.jp

A. Taniguchi

e-mail: amtanigu@ior.twmu.ac.jp

S. Momohara

e-mail: smomohara@ior.twmu.ac.jp

N. Sugiyama

Medical Affairs, Pfizer Japan Inc., Shinjuku Bunka Quint Bldg.,

3-22-7 Yoyogi, Shibuya-ku, Tokyo 151-8589, Japan

e-mail: naonobu.sugiyama@pfizer.com
Methotrexate was used by $27 \%$ of patients. In 2005, $5 \%$ of patients were prescribed methotrexate at $>8 \mathrm{mg} / \mathrm{week}$, which increased to $13 \%$ in 2011. These rates were lower than those in the IORRA cohort.

Conclusions Our results indicate that the prevalence of RA in Japan is somewhere between 0.6 and $1.0 \%$. Considering that methotrexate is infrequently used, the implementation of aggressive treatment regimens such as the 'Treat to Target' strategy is important to achieve tight control of RA in Japan.

Keywords Prevalence $\cdot$ Rheumatoid arthritis - Database · Health insurance $\cdot$ Epidemiology

\section{Introduction}

The treatment of rheumatoid arthritis (RA) has advanced markedly over the last 10 years [1]. The introduction of methotrexate (MTX), the foundation for RA treatment, and biological products targeting specific molecules that induce or aggravate the inflammation associated with RA, have increased the likelihood of achieving clinical remission, and potentially structural and functional remission.

In Japan, MTX was approved for the treatment of RA in 1999, some 10 years later than its approval in the United States (US) and Europe. At that time, the dose of MTX was limited to $8 \mathrm{mg} /$ week. In addition, MTX was not originally approved as a first-line treatment in Japan. Consequently, MTX has been underused for many years in Japan. In 2011, the Ministry of Health, Labour and Welfare approved MTX doses of up to $16 \mathrm{mg} /$ week and its use as a first-line drug, partly based on actual treatment practices [2]. MTX is now widely recommended as a foundation of RA treatment in internationally recognized treatment guidelines [3, 4]. 
Of concern, however, is that relatively few epidemiological studies aimed at determining the prevalence of and treatment practices for RA have been performed in Japan [5-7], unlike in the US and Europe. The studies performed to date focused on specific hospitals or on a specific region of Japan, and did not examine the nationwide prevalence of RA in Japan. Therefore, the true prevalence of RA in Japan has not been adequately investigated. In addition, despite recent advances in treatment options for RA, there have been no nationwide studies aimed at examining the adoption of the newer treatments in actual clinical practice, including patients under the care of non-specialists, although some cohort studies have been conducted in limited medical institutions [2, 8-11].

Therefore, we conducted a study with the following aims: (1) to determine the current prevalence of RA in Japan and (2) to examine current treatment practices. To achieve these aims, we conducted analyses of reimbursement data covering $\sim 1$ million people $(0.84 \%$ of the Japanese population) who were members of Japanese health insurance societies. In this study, we determined the estimated prevalence of RA in Japan and examined the changes in treatment patterns over time, particularly of MTX, which is recommended as the anchor drug in the current treatment strategies for RA. We also compared the treatment patterns identified in this reimbursement database with those of the Institute of Rheumatology, Rheumatoid Arthritis (IORRA) cohort, which is managed by Tokyo Women's Medical University [1, 2, 8-11]. The objective of this comparison with the IORRA cohort was to determine whether the treatment practices applied in general clinical practice are similar to those applied in a specialist center focusing on RA.

\section{Methods}

\section{Study database}

This analysis was conducted using reimbursement data provided by the Japan Medical Data Center Co., Ltd. (JMDC) [12], which was purchased by Pfizer, Japan. JMDC, through contracts with multiple Japanese health insurance societies (as of October 2012, there are 20 health insurance societies contributing to the JMDC database), has accumulated reimbursement data from 1,067,782 people, including healthy individuals aged $<75$ years. In Japan, there is a separate medical insurance system for individuals aged $\geq 75$ years; thus, when patients reach the age of 75 years, they are automatically removed from health insurance societies. As a result, the JMDC database only includes patients aged $<75$ years and patients within 1 month of their 75th birthday; therefore, the number of patients aged 75 years or older in the database is very low. In this database, personal information is encrypted irreversibly, information is recorded under patient names, and medical consultation information can be tracked for each patient in a chronological order, even if the patient attends multiple medical institutions or following hospital transfers. Wherever possible, diagnoses are recorded in the JMDC database using International Classification of Diseases (ICD) version 10 codes. Considering its size, representativeness for Japan, and the scope of data recorded, it is possible to use the data to estimate the national incidence of a specific disease. JMDC started collecting data for 320 thousand people in 2005, which increased to 600 thousand people from 2008, to 750 thousand people in 2009, and to 1 million people in 2010. All patients are continuously tracked in the database, which contains data for up to 7 years, as of January 2012, depending on when each patient was first registered. In this analysis, we used data registered in 2005 and 2010.

The IORRA cohort is a prospective observational cohort of RA patients treated at the Institute of Rheumatology, Tokyo Women's Medical University from October 2000 [1, 2, 8-11]. Patients with RA who fulfilled the American College of Rheumatology 1987 criteria for RA [13] were registered, and their information and data were collected biannually (in April and October). Informed consent was obtained from each patient at each visit. Over $98 \%$ of the RA patients attending our institute participated in the study each time. For the purpose of this study, we used data registered between April 2005 and October 2011 to match the period recorded by the reimbursement database. The distribution of patients aged $\geq 75$ years in the IORRA cohort was low, and ranged from $9.4 \%$ in April, 2005, to $12.3 \%$ in October, 2011. To allow for direct comparisons between the JMDC data and the IORRA cohort, we limited the analyses of the IORRA cohort to patients aged $<75$ years.

Estimation of the prevalence of RA and prescription rates of drugs for RA

Using reimbursement data for 1 million people registered in 2010, we estimated the prevalence of RA and the number of patients prescribed any drugs for RA. We then estimated the prevalence of RA and drug prescription for the period July 2010 to June 2011. The diagnosis of RA and suspected RA was based on the disease name stated on health insurance claim forms, as recorded in the database using ICD 10 codes (M05.9, RA with a positive serological reaction, detail unknown; M06.0, RA with a negative serological reaction; M06.8, RA specified in other types; M06.9, RA, detail unknown). Using the JMDC database, the prevalence of RA was estimated by calculating the proportion of patients with a confirmed or suspected 
Table 1 Treatments available for rheumatoid arthritis in Japan

\begin{tabular}{|c|c|}
\hline Drug class & Drugs available and ATC code, where applicable \\
\hline Oral NSAIDs & $\begin{array}{l}\text { Anti-inflammatory and antirheumatic products, non- } \\
\text { steroids }[\mathrm{M} 01 \mathrm{~A}]\end{array}$ \\
\hline $\begin{array}{l}\text { NSAID } \\
\text { patches }\end{array}$ & Topical products for joint and muscular pain [M02] \\
\hline \multirow[t]{2}{*}{ Oral steroids } & Corticosteroids for systemic use, plain [H02A2] \\
\hline & $\begin{array}{l}\text { Corticosteroids for systemic use, combinations } \\
\text { [H02B] }\end{array}$ \\
\hline DMARDs & $\begin{array}{l}\text { Actarit, auranofin, salazosulfapyridine, bucillamine, } \\
\text { lobenzarit disodium, sodium aurothiomalate, } \\
\text { D-penicillamine, tacrolimus, mizoribine and } \\
\text { leflunomide }\end{array}$ \\
\hline MTX & MTX [M01C] \\
\hline $\begin{array}{r}\text { Biological } \\
\text { products }\end{array}$ & $\begin{array}{l}\text { Tocilizumab, adalimumab, infliximab, etanercept } \\
\text { and abatacept }\end{array}$ \\
\hline
\end{tabular}

ATC anatomical therapeutic chemical, NSAID non-steroidal antiinflammatory drug, DMARD disease-modifying antirheumatic drugs, MTX methotrexate

diagnosis of RA for sex and 1-year age groups. We then multiplied the obtained values by the number of males or females recorded in the Japanese Population Census (Statistics Bureau, Ministry of Internal Affairs and Communications; http://www.e-stat.go.jp/SG1/estat/GL08020103.do? _xlsDownload_\&fileId=000005147291\&releaseCount=2) for each 1-year age group (total population, 128 million). Since the study population consisted of subscribers to health insurance societies, and included relatively few elderly individuals, this analysis was limited to patients aged $\geq 16$ to $<75$ years. We excluded patients aged $<16$ years because they should be diagnosed with juvenile idiopathic arthritis (JIA) rather than rheumatoid arthritis. Drugs used to treat RA included oral NSAIDs (non-steroidal antiinflammatory drugs), NSAID patches and oral steroids, and drugs listed in the anatomical therapeutic chemical (ATC) classification system were identified from the reimbursement data. Regarding MTX, only the brand (i.e., Rheumatrex) and generic products specifically indicated for the treatment of RA, class M01C, were identified (Table 1). As a sensitivity analysis we excluded patients using NSAID patches alone and patients prescribed other drugs only once.

Changes over time in the prescription of drugs for RA

The prevalence of RA from January 2005 to June 2011 was calculated using data for 320 thousand individuals aged $<75$ years. Then, the proportions of patients prescribed any drugs for RA and with specific drugs were tabulated for every 6-month period. The values recorded in both 6-month periods in the preceding year were used to examine the changes in prescription rates over time. The proportion of patients prescribed MTX of all patients prescribed diseasemodifying anti-rheumatic drugs (DMARDs), including MTX, was also determined. In the IORRA cohort, the drugs that the patients actually took in the 6 months before each visit were reported by each patient using questionnaires.

Changes over time in the dose of MTX

The number of prescriptions for each dose level of MTX between January 2005 and June 2011 was tabulated using data for 320 thousand people for every half year, and values for the preceding 1 year were calculated to examine changes over time. Since the medical receipts did not always specify MTX dose, or the dose was unclear, only prescription receipts were analyzed. The changes in MTX dose over time were based on the actual doses prescribed rather than the number of patients with RA. For example, a patient with three individual prescriptions of three different doses of MTX (e.g., 6, 8 and $10 \mathrm{mg} /$ week MTX) was included in three separate analyses, one for each dose. The data from prescription sheets lacking the required dose (including those with an unclear dose) were not recorded in the JMDC database. We did not restrict this analysis by age. In the IORRA cohort, the MTX dose taken by the patient in the 6 months before each visit was reported by each patient using questionnaires.

\section{Results}

Prevalence of and treatment practices for RA in Japan

The estimated prevalence of RA from July 2010 to June 2011 relative to the total population of Japan aged $<75$ years was determined based on the disease names recorded on health insurance claim forms and recent census data. The total number of RA patients aged $\geq 16$ to $<75$ years old (excluding suspected RA) was 5,344 (3,868 females; 1,476 males) (Supplemental Table). The results of this analysis, and types of treatments used, are summarized in Table 2. The number of RA patients was estimated as 2.29 million if suspected cases are included; as a sensitivity analysis, if suspected cases were excluded, the number of RA patients was estimated as 1.24 million. Thus, the estimated prevalence of RA was $1.0 \%$ of the entire Japanese population aged $\geq 16$ to $<75$ years.

Overall, three times as many females as males were diagnosed with RA (913 thousand, $74 \%$ vs 328 thousand, $26 \%$, of 1.24 million). The age distribution was as follows: $\geq 16$ to $<20$ years: $0.3 \%, 4$ thousand; 20 to $<30$ years: $1.5 \%, 18$ thousand; 30 to $<40$ years: $5.2 \%, 65$ thousand; 40 to $<50$ years: $9.7 \%, 121$ thousand; 50 to $<60$ years: $22.5 \%$, 279 thousand; 60 to $<70$ years: $43.3 \%, 538$ thousand; and 70-74 years: $17.5 \%, 217$ thousand. The estimated number 
Table 2 Estimated prevalence of RA in Japan

\begin{tabular}{|c|c|c|c|c|}
\hline & $\begin{array}{l}\text { Estimate } \\
(\times 1,000)\end{array}$ & $\begin{array}{l}95 \% \text { CI } \\
(\times 1,000)\end{array}$ & Composite $1^{\mathrm{a}}(\%)$ & Composite $2^{\mathrm{b}}(\%)$ \\
\hline \multicolumn{5}{|l|}{ Prevalence of $\mathrm{RA}^{\mathrm{c}}$} \\
\hline Including suspected RA & 2,287 & $2,224-2,367$ & - & - \\
\hline Excluding suspected RA & 1,241 & $1,194-1,306$ & 100 & - \\
\hline \multicolumn{5}{|l|}{ Treatments received $^{\mathrm{d}}$} \\
\hline Any drug & 983 & $941-1,043$ & 79 & 100 \\
\hline Oral NSAIDs & 681 & $646-734$ & 55 & 69 \\
\hline NSAID patches & 580 & $548-630$ & 47 & 59 \\
\hline Oral steroids & 371 & $346-417$ & 30 & 38 \\
\hline DMARDs (including MTX) & 500 & $471-548$ & 40 & 51 \\
\hline DMARDs (excluding MTX) & 266 & $245-306$ & 21 & 27 \\
\hline MTX & 329 & $308-371$ & 27 & 34 \\
\hline Biological products & 95 & $86-125$ & 8 & 10 \\
\hline
\end{tabular}

$R A$ rheumatoid arthritis, $C I$ confidence interval, NSAID non-steroidal anti-inflammatory drug, MTX methotrexate

${ }^{a}$ Of all patients with documented RA

${ }^{\mathrm{b}}$ Of all patients on treatment for RA

c Estimates were based on the disease name recorded on health insurance claim forms

${ }^{d}$ Estimates were based on the drugs stated on health insurance claim forms

of patients with RA and under treatment for this condition between July 2010 and June 2011 was 983 thousand.

In terms of treatment, $79 \%$ of all patients with RA (excluding suspected RA) had been prescribed a drug for RA during the indicated period. The most commonly prescribed drug classes in this population were oral NSAIDs (55\% of patients), NSAID patches (47\%), DMARDs (including MTX; $40 \%$ ) and oral steroids (30\%). MTX was prescribed to $27 \%$ of patients and only $8 \%$ were prescribed biological products. As a sensitivity analysis, we excluded patients using NSAID patches alone. Among patients with a definitive diagnosis of RA who were prescribed a drug only once (defined as a prescription filled in just 1 month in the 12-month analysis period), $8 \%$ were using NSAID patches alone and $14 \%$ of patients were prescribed drugs other than NSAID patches (oral NSAIDs only, $10 \%$; oral steroids only, $1 \%$; oral NSAIDs and oral steroids, $1 \%$; and other medications, $2 \%$ ). For the purpose of this analysis, we used the following assumption. Subtracting the $8 \%$ of patients prescribed only NSAID patches and $14 \%$ of patients prescribed other drugs for only 1 month from the $79 \%$ of patients treated with any drug, left $57 \%$ of the patients (706 thousand patients) or approximately $0.6 \%$ of the Japanese population.

Changes in the prescribing practices for drugs for RA over time

We next determined changes in the prescription rates of drugs for RA. The results shown in Fig. 1a and b indicate that the prescription of biological products and MTX increased over time, whereas no marked changes were observed for the other drugs for RA. As shown in Fig. 2a, the proportion of patients using MTX of all patients prescribed a DMARD increased from $34 \%$ in December 2005 to $63 \%$ in June 2011, indicating increased reliance on MTX as a DMARD in Japan.

The prescription rates of MTX and biological products also increased in the IORRA cohort (Fig. 1c), although both were used more frequently in the IORRA cohort than in the reimbursement database. The proportion of patients using MTX of all patients prescribed a DMARD increased substantially, from $64 \%$ in 2005 to $83 \%$ in 2011 (Fig. 2b). Another difference between the IORRA cohort and the reimbursement database is that the use of oral steroids, oral NSAIDs, and DMARDs (excluding MTX) decreased over time (Fig. 1c).

\section{Changes in MTX doses over time}

Until 2011, the maximum approved dose of MTX in Japan was $8 \mathrm{mg} /$ week. As shown in Fig. 3a, at the start of data collection in 2005, $\sim 95 \%$ of patients were being prescribed MTX at doses of $\leq 8 \mathrm{mg} /$ week, while $5 \%$ were receiving doses $>8 \mathrm{mg} /$ week. The prescription rates of high doses of MTX have increased progressively over time; as of June 2011, $13 \%$ were receiving doses $>8 \mathrm{mg} /$ week. An increase in MTX dose was also evident in the IORRA cohort, although the dose was much higher (Fig. 3b). In the IORRA cohort, the proportion of patients 

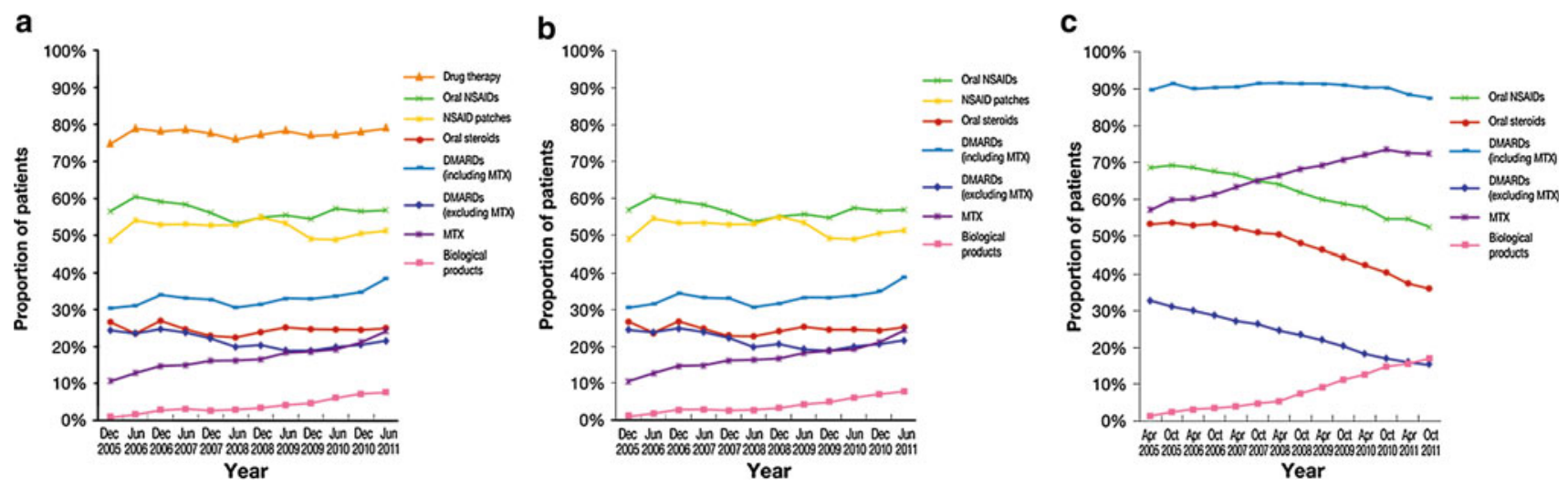

Fig. 1 Changes over time in the proportion of patients prescribed specific classes of drugs for RA. a Of all patients diagnosed with RA. b Of all patients being treated for RA. c Results of the IORRA cohort.

The number of patients aged $<75$ years in the IORRA cohort ranged from 4,312 in 2005 to 5,001 in 2011
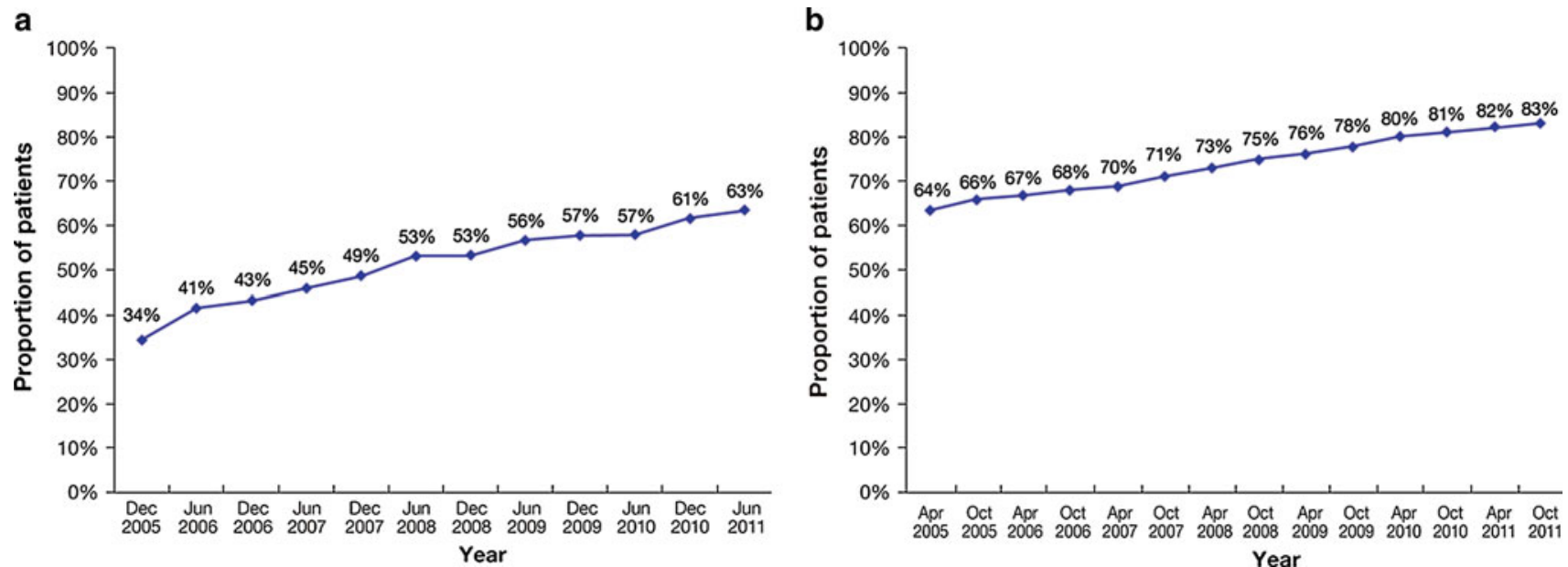

Fig. 2 a Proportion of patients receiving methotrexate of all patients treated with disease-modifying antirheumatic drugs. b Results of the IORRA cohort. The number of patients aged $<75$ years in the IORRA cohort ranged from 4,312 in 2005 to 5,001 in 2011

taking MTX at a dose $>8 \mathrm{mg} /$ week was $20 \%$ in 2005 and $42 \%$ in 2011.

\section{Discussion}

Health insurance databases, which have been established using reimbursement claims data, provide a powerful tool for health-economic analyses that reflect daily clinical practice in the target population [14-16]. In the US and Europe, numerous studies of health insurance subscribers have already been conducted, focusing on RA [17-22]. These studies have analyzed the proportion of patients receiving specific drugs, the doses used, treatment continuation rates, and medical expenses [23, 24]. In Japan, similar studies have been conducted in the fields of osteoporosis [25] and Parkinson's disease [26]. We think that such analyses will become increasingly common, and will help to better understand current clinical practice in Japan and in other countries. Therefore, we took advantage of the large sample size of the health insurance database to estimate the prevalence and treatment patterns of RA in Japan.

In this analysis, the number of patients diagnosed with RA based on prescriptions was 1.24 million and the number of patients prescribed anti-RA drugs was 983 thousand. After subtracting the number of patients prescribed only NSAID patches and those prescribed drugs other than NSAID patches only once, we were left with 706 thousand patients. The values of 1.24 million, 983 thousand, and 706 thousand correspond to $1.0,0.8$, and $0.6 \%$ of the Japanese population, respectively.

In early studies that did not include age limits, The Ministry of Health, Labor and Welfare of Japan [27] estimated that there were 336 thousand individuals with RA who were continuously receiving medical care at clinics or hospitals. Shichikawa et al. [6] estimated the prevalence of 

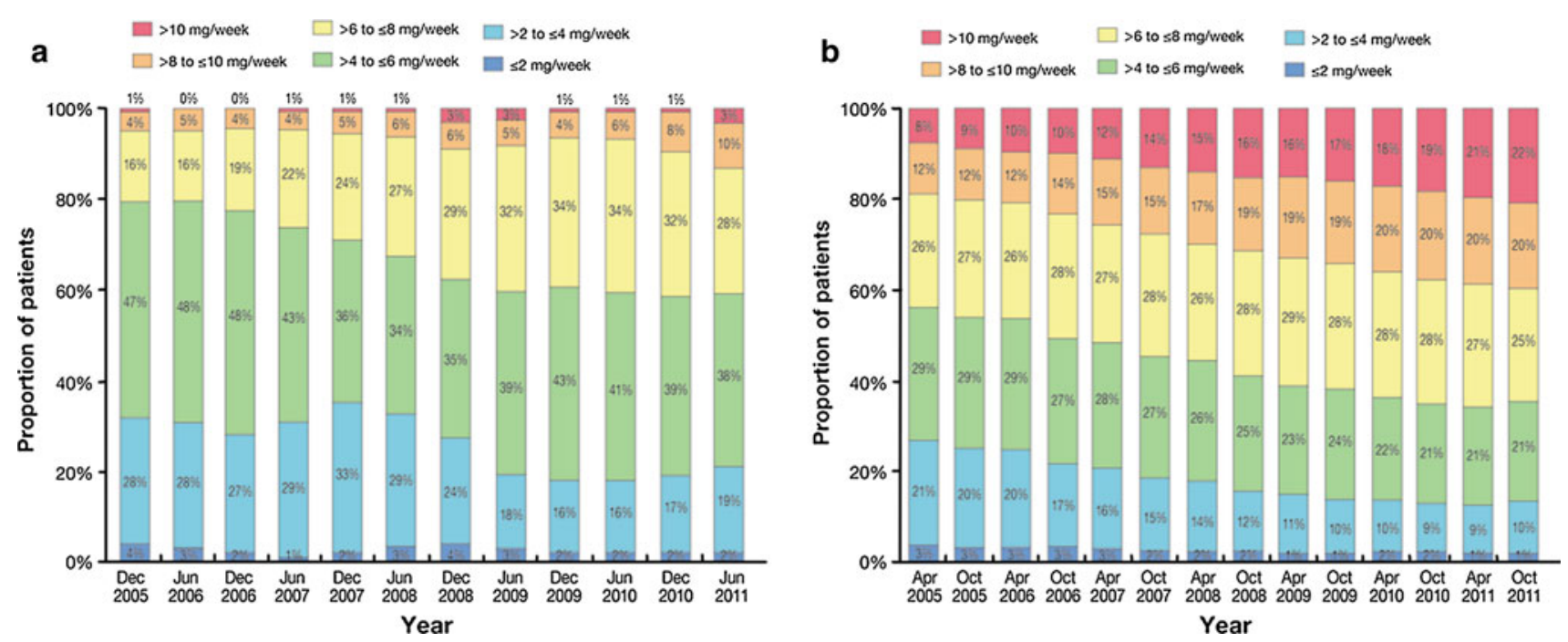

Fig. 3 a Changes in methotrexate dose over time. b Results of the IORRA cohort. The number of patients in the IORRA cohort ranged from 4,312 in 2005 to 5,001 in 2011

RA in Wakayama Prefecture, Japan, and reported that it reached a peak in $1969(0.54 \%, 95 \%$ CI $0.17-0.87 \%)$ and was lowest in $1996(0.17 \%, 95 \%$ CI $0.03-0.31 \%)$. Meanwhile, the estimated prevalence of RA ranged from 0.5 to $1 \%$ in a systematic review of studies conducted in Western countries [28] including the US $(1.07 \%)$ and northern Europe $(0.50 \%, 95 \%$ CI 0.44-0.80 \%), although slightly lower rates were reported in southern Europe $(0.33 \%, 95 \%$ CI $0.31-0.50 \%)$ and in developing countries $(0.35 \%, 95 \%$ CI $0.24-0.36 \%)$. Although the estimated prevalence of $0.6 \%$ in our study is a conservative estimate and was limited to individuals aged $\geq 16$ to $<75$ years, it corresponds to the upper limit in Japan and lower limits in Western countries. Therefore, we might expect the prevalence rate to be slightly higher than that reported in earlier studies.

However, we should consider that methodological differences (e.g., analysis of health insurance/reimbursement data and epidemiological questionnaires using randomized, clustered samples) prevent direct comparisons of the results of the studies described above.

The second objective of this study was to examine the current status of RA treatment in Japan, and changes in recent years. Over the past 10 years, several biological products have been introduced into clinical settings, and the 'Treat to Target' approach is increasingly being adopted since its inception in 2010 [1,29]. As a result, there is increasing demand for achieving tight control of the disease, requiring aggressive treatment strategies, as well as changes in the regulatory environment. Moreover, since MTX, which is the anchor drug for RA treatment, is now indicated for use as a first-line treatment for RA, and the maximum approved dose has been increased to $16 \mathrm{mg}$, it seems likely that RA treatment has undergone marked changes in recent years. Changes in RA treatment practices in Japan have been evaluated by several research groups, including the IORRA cohort managed by the Institute of Rheumatology, Tokyo Women's Medical University [1, 11]. In that study, as in the present study, the proportions of patients treated with MTX and biological products, have steadily increased over time. However, as of June 2011 [1], $70 \%$ of patients were receiving MTX, of which $38 \%$ received doses exceeding $8 \mathrm{mg} / \mathrm{week}$. The proportion of patients receiving biological products was $15 \%$ [1]. Notably, those values were higher than those obtained in the present study. However, this may be due to differences in data collection. In the present study, doses were extracted from a health insurance database, which recorded the number of prescriptions and the prescribed doses. By comparison, in the IORRA study, patients kept a record of the actual doses taken, which also allowed the authors to assess compliance rates. Therefore, the results of the two studies should not be compared directly. One may expect the actual doses to be taken to be smaller than the prescribed doses, resulting in a bias toward a smaller difference in dose between the two studies. Considering that the differences were quite substantial, we think that aggressive treatment strategies for RA are being adopted in medical institutions specialized for treating RA, such as Tokyo Women's Medical University, but less so in general clinical practice. In this context, it is interesting to see that the use of oral steroids, oral NSAIDs, and DMARDs, other than MTX, has decreased over time in the IORRA cohort but not in the reimbursement database. These results 
suggest that nationwide educational programs are needed to increase awareness and implementation of recent treatment strategies in Japan.

The results of this study should be interpreted with some caution, taking into account the limitations of the study. We analyzed a database of 1 million people belonging to health insurance societies in Japan. Therefore, the database may not fully represent the general population of Japan, as elderly individuals are less likely to be members of such societies. Considering the database includes relatively few elderly patients, the treatment situation described here may not reflect that of actual clinical practice. Meanwhile, relatively few younger patients were diagnosed with RA using this database, most likely reflecting the higher incidence of JIA diagnosis rather than RA itself. It is also possible that some of the patients aged $<20$ years with RA actually had JIA, which may result in an overestimation of the prevalence of RA. However, as there were only 45 patients with RA aged $<20$ years the impact of this is likely to be minimal. Nevertheless, to minimize the impact of this, we excluded patients aged $<16$ years from the analysis, as this age-group is more likely to have JIA. Finally, the 'quality' of diagnosis may be a limiting factor, as some patients were 'diagnosed' with RA in this study on the basis of diagnosis written in claim forms, while other patients were assumed to have RA on the basis of prescription for drugs that are routinely used for RA. However, as $8 \%$ of patients were only prescribed NSAID patches and $14 \%$ of patients received other drugs for only 1 month, it is possible that these patients were given a nominal diagnosis of RA so that they could be prescribed drugs specific for RA. To help track disease names and diagnoses, all diseases were recorded in the database using ICD-10 codes, which were used wherever possible.

It is important to consider that the designs of various studies may limit direct comparisons. Generally, the prevalence of a disease such as RA is ascertained either by surveys of patients or through analysis of health insurance/ reimbursement data. Patient surveys assess the patient's main disease/injury, without taking into account complications. Furthermore, such studies may result in bias towards patients under active treatment; patients with low disease activity, other more serious diseases, or cognitive limitations, for example, are less likely to respond to such surveys [30]. Additionally, such studies may involve relatively small numbers of patients identified through clustered sampling. Considering these limitations of traditional patient surveys, there is an increasing shift towards analyses of larger databases managed by public and private health insurance providers. These databases, including the JMDC, can record data for a much larger cohort of patients than is generally possible with patient surveys, along with longitudinal data for individual patients. Additionally, the databases can record information on specific diagnoses, treatments prescribed, duration of treatment, and changes in prescribing behavior over time [14, 15]. The resulting data can be used in the context of diagnostic, etiologic, prognostic, and intervention research [14, 15], as well as aiding coverage and reimbursement decisions [16]. As described above, however, health insurance databases may be subject to other forms of bias. In particular, private health insurance societies may include a relatively small number of elderly patients, which may result in an underestimation of diseases that predominantly affect elderly individuals, such as RA. In addition, some drugs may be prescribed for off-label uses. Direct assessment of comorbidities may also be difficult using health insurance databases [31]. Therefore, care must be taken when assessing diseases based on prescribed drugs alone.

In conclusion, the current study revealed that the prevalence of RA in Japan is quite high (1.0\%, 1.24 million individuals aged $\geq 16$ to $<75$ years). Based on this result and other sensitivity analyses, we suggest that the prevalence of RA in Japan is somewhere between 0.6 and $1.0 \%$. The use of MTX doses $>8 \mathrm{mg} /$ week and biological products is steadily increasing, consistent with the shift towards aggressive treatment paradigms. However, the rates are still quite low, suggesting that educational programs are needed to raise awareness of the need for aggressive treatment regimens, such as the 'Treat to Target' strategy, to improve outcomes of Japanese patients with RA.

Conflict of interest The IORRA cohort was supported by nonrestricted research grants from 36 pharmaceutical companies: Abbott Japan, Asahi Kasei Kuraray Medical, Asahi Kasei Pharma, Astellas Pharma, AstraZeneca, MSD, Chugai Pharmaceutical, Daiichi Fine Chemical, Daiichi Sankyo, Dainippon Sumitomo Pharma, Eisai, GlaxoSmithKline, Janssen Pharmaceutical, Japan Tobacco, Kaken Pharmaceutical, Kissei Pharmaceutical, Kowa Pharmaceutical, Mitsubishi Chemical Medience, Mitsubishi Tanabe Pharma, Nippon Chemiphar, Nippon Shinyaku, Novartis Pharma, Otsuka Pharmaceutical, Pfizer Japan, Sanofi-Aventis, Santen Pharmaceutical, Sanwa Kagaku Kenkyusho, Sekisui Medical, Taisho Toyama Pharmaceutical, Takeda Pharmaceutical, Teijin Pharma, Torii Pharmaceutical, Toyama Chemical, UCB Japan, Pfizer, Zeria Pharmaceutical. H. Yamanaka has received consultancy fees, speaking fees, and/or honoraria from Chugai Pharmaceutical, Daiichi Sankyo, Mitsubishi Tanabe Pharma, Abbott Japan, Eisai, Takeda Pharmaceutical, Janssen Pharmaceutical, Hoffmann-La Roche, and Pfizer. S. Momohara has received speaking fees from Chugai Pharmaceutical, Eisai, and Mitsubishi Tanabe Pharma. N. Sugiyama is an employee of Pfizer Japan Inc.

\section{References}

1. Yamanaka H, Seto Y, Tanaka E, Furuya T, Nakajima A, Ikari K, et al. Management of rheumatoid arthritis: the 2012 perspective. Mod Rheumatol. 2012. doi:10.1007/s10165-012-0702-1.

2. Seto Y, Tanaka E, Inoue E, Nakajima A, Taniguchi A, Momohara $\mathrm{S}$, et al. Studies of the efficacy and safety of methotrexate at 
dosages over $8 \mathrm{mg} /$ week using the IORRA cohort database. Mod Rheumatol. 2011;21:579-93. doi:10.1007/s10165-011-0445-4.

3. Singh JA, Furst DE, Bharat A, Curtis JR, Kavanaugh AF, Kremer JM, et al. 2012 update of the 2008 American College of Rheumatology recommendations for the use of disease-modifying antirheumatic drugs and biologic agents in the treatment of rheumatoid arthritis. Arthritis Care Res (Hoboken). 2012;64: 625-39. doi:10.1002/acr.21641.

4. Smolen JS, Landewe R, Breedveld FC, Dougados M, Emery P, Gaujoux-Viala C, et al. EULAR recommendations for the management of rheumatoid arthritis with synthetic and biological disease-modifying antirheumatic drugs. Ann Rheum Dis. 2010;69:964-75. doi:10.1136/ard.2009.126532.

5. Imanaka T, Shichikawa K, Inoue K, Shimaoka Y, Takenaka Y, Wakitani S. Increase in age at onset of rheumatoid arthritis in Japan over a 30-year period. Ann Rheum Dis. 1997;56:313-6.

6. Shichikawa K, Inoue K, Hirota S, Maeda A, Ota H, Kimura M, et al. Changes in the incidence and prevalence of rheumatoid arthritis in Kamitonda, Wakayama, Japan, 1965-1996. Ann Rheum Dis. 1999;58:751-6.

7. Watanabe K, Urata S, Suzuki K, Ohba M, Inagaki Y. Progressive and nonprogressive rheumatoid arthritis over a 10-year period in Japan. J Rheumatol Suppl. 2004;69:27-9.

8. Momohara S, Inoue E, Ikari K, Yano K, Tokita A, Honjo Y, et al. Comparison of characteristics and therapeutic efficacy in rheumatoid arthritis patients treated by rheumatologists and those treated by orthopedic surgeons under a team medicine approach at the same institute. Mod Rheumatol. 2012;22:209-15. doi: 10.1007/s10165-011-0495-7.

9. Nakajima A, Inoue E, Shidara K, Hoshi D, Sato E, Seto Y, et al. Standard treatment in daily clinical practice for early rheumatoid arthritis improved disease activity from 2001 to 2006. Mod Rheumatol. 2011;21:594-7. doi:10.1007/s10165-011-0457-0.

10. Yamanaka H. A cohort study of clinical care in rheumatoid arthritis: the IORRA study. JMAJ. 2009;52:54-6.

11. Yamanaka H, Inoue E, Singh G, Tanaka E, Nakajima A, Taniguchi A, et al. Improvement of disease activity of rheumatoid arthritis patients from 2000 to 2006 in a large observational cohort study IORRA in Japan. Mod Rheumatol. 2007;17:283-9. doi:10.1007/s10165-007-0587-6.

12. Kimura S, Sato T, Ikeda S, Noda M, Nakayama T. Development of a database of health insurance claims: standardization of disease classifications and anonymous record linkage. J Epidemiol. 2010;20:413-9.

13. Arnett FC, Edworthy SM, Bloch DA, McShane DJ, Fries JF, Cooper NS, et al. The American Rheumatism Association 1987 revised criteria for the classification of rheumatoid arthritis. Arthritis Rheum. 1988;31:315-24.

14. Martin-Latry K, Begaud B. Pharmacoepidemiological research using French reimbursement databases: yes we can! Pharmacoepidemiol Drug Saf. 2010;19:256-65. doi:10.1002/pds.1912.

15. Smeets HM, de Wit NJ, Hoes AW. Routine health insurance data for scientific research: potential and limitations of the Agis Health Database. J Clin Epidemiol. 2011;64:424-30. doi: 10.1016/j.jclinepi.2010.04.023.

16. Garrison LP Jr, Neumann PJ, Erickson P, Marshall D, Mullins CD. Using real-world data for coverage and payment decisions: the ISPOR Real-World Data Task Force report. Value Health. 2007;10:326-35. doi:10.1111/j.1524-4733.2007.00186.x.

17. Bernard MA, Benichou J, Blin P, Weill A, Begaud B, Abouelfath $A$, et al. Use of health insurance claim patterns to identify patients using nonsteroidal anti-inflammatory drugs for rheumatoid arthritis. Pharmacoepidemiol Drug Saf. 2012;. doi:10.1002/pds.3221.

18. Cannon GW, Mikuls TR, Hayden CL, Ying J, Curtis JR, Reimold AM, et al. Merging Veterans Affairs rheumatoid arthritis registry and pharmacy data to assess methotrexate adherence and disease activity in clinical practice. Arthritis Care Res (Hoboken). 2011;63:1680-90. doi:10.1002/acr.20629.

19. McBride S, Sarsour K, White LA, Nelson DR, Chawla AJ, Johnston JA. Biologic disease-modifying drug treatment patterns and associated costs for patients with rheumatoid arthritis. J Rheumatol. 2011;38:2141-9. doi:10.3899/jrheum.101195.

20. Petri H, Maldonato D, Robinson NJ. Data-driven identification of co-morbidities associated with rheumatoid arthritis in a large US health plan claims database. BMC Musculoskelet Disord. 2010; 11:247. doi:10.1186/1471-2474-11-247.

21. Thayer S, Watson C, Song R, Globe DR, Harrison DJ. Etanercept treatment patterns in managed-care patients with psoriasis or psoriatic arthritis. J Med Econ. 2010;13:228-35. doi:10.3111/ 13696998.2010.487469.

22. Wong BJ, Cifaldi MA, Roy S, Skonieczny DC, Stavrakas S. Analysis of drug and administrative costs allowed by US private and public third-party payers for 3 intravenous biologic agents for rheumatoid arthritis. J Manag Care Pharm. 2011;17:313-20.

23. Harley CR, Frytak JR, Tandon N. Treatment compliance and dosage administration among rheumatoid arthritis patients receiving infliximab, etanercept, or methotrexate. Am J Manag Care. 2003;9:S136-43.

24. Yazici Y, Krasnokutsky S, Barnes JP, Hines PL, Wang J, Rosenblatt L. Changing patterns of tumor necrosis factor inhibitor use in 9,074 patients with rheumatoid arthritis. J Rheumatol. 2009; 36:907-13. doi:10.3899/jrheum.080592.

25. Kirigaya D, Nakayama $T$, Ishizaki $T$, Ikeda $S$, Satoh $T$. Management and treatment of osteoporosis in patients receiving long-term glucocorticoid treatment: current status of adherence to clinical guidelines and related factors. Intern Med. 2011;50: 2793-800.

26. Nakaoka S, Ishizaki T, Urushihara H, Satoh T, Ikeda S, Morikawa K, et al. Echocardiography for the detection of valvulopathy associated with the use of ergot-derived dopamine agonists in patients with Parkinson's disease. Intern Med. 2011;50:687-94.

27. Ministry of Health, Labour and Welfare. Results of the 2008 patient survey: Total number of patients with severe disease. http://www.mhlw.go.jp/toukei/saikin/hw/kanja/08/dl/05.pdf (In Japanese). Last Accessed 10 August 2012.

28. Alamanos Y, Voulgari PV, Drosos AA. Incidence and prevalence of rheumatoid arthritis, based on the 1987 American College of Rheumatology criteria: a systematic review. Semin Arthritis Rheum. 2006;36:182-8. doi:10.1016/j.semarthrit.2006.08.006.

29. Smolen JS, Aletaha D, Bijlsma JW, Breedveld FC, Boumpas D, Burmester G, et al. Treating rheumatoid arthritis to target: recommendations of an international task force. Ann Rheum Dis. 2010;69:631-7. doi:10.1136/ard.2009.123919.

30. Gayet-Ageron A, Agoritsas T, Schiesari L, Kolly V, Perneger TV. Barriers to participation in a patient satisfaction survey: who are we missing? PLoS ONE. 2011;6:e26852. doi:10.1371/journal. pone. 0026852

31. Klabunde CN, Warren JL, Legler JM. Assessing comorbidity using claims data: an overview. Med Care. 2002;40:IV-26-35. doi:10.1097/01.mlr.0000020936.03651.2d. 